\title{
Dilated Virchow-Robin Space and Dopamine Transporter Imaging in the Striatum of Patients with Parkinsonism
}

\author{
Dokyung Lee, Il Ki Hong, Tae-Beom Ahn
}

\begin{abstract}
Objective: The radiological and clinical significance of a dilated Virchow-Robin space (dVRS) in the striatum (STR) remains unclear. We investigated the role of dVRS in STR on parkinsonism and dopamine transporter positron emission tomography (DaT-PET) findings. Methods: Patients with parkinsonism who underwent both brain magnetic resonance imaging and DaT-PET were included. Clinical status was evaluated by Hoehn and Yahr (HY) stage, Korean-Mini Mental Status Examination (K-MMSE), Montreal Cognitive Assessment Korea (MoCA-K), and Frontal Assessment Battery (FAB). dVRS was assessed by semi-quantitative and quantitative scales in each of the three segments of STR (caudate nuclei, anterior and posterior putamen) and was expressed as a dVRS score. DaT-PET was qualitatively assessed as either normal or abnormal in each segment. The relationship between dVRS and DaT-PET abnormality (ab-DaT-PET) was designated in each segment as either concordant or discordant. A concordant segment was defined by the presence of dVRS with ab-DaT-PET [Concordance rate $(\mathrm{CR})=$ number of concordant segments/number of concordant and discordant segments]. Results: Eleven patients were included. There was no significant correlation between the presence of dVRS and ab-DaT-PET. The mean CR was 0.39 . CR was not significantly correlated with any clinical or neuroimaging scales. The dVRS score was significantly correlated with K-MMSE, MoCA-K, and FAB ( $r=-0.675,-0.847$, and -0.868 , respectively) but not with HY stage. Conclusion: dVRS in STR played no significant role on dopaminergic innervation revealed by DaT-PET and made little contribution to clinical parkinsonism; however, it was correlated with cognitive impairment.
\end{abstract}

RÉSUMÉ: Dilatation de l'espace périvasculaire de Virchow-Robin et imagerie du transporteur de la dopamine dans le striatum chez des patients atteints de parkinsonisme. Objectif: La signification radiologique et clinique d'un espace de Virchow-Robin dilaté (EVRd) dans le striatum n'est pas encore élucidée. Nous avons étudié le rôle d'un EVRd dans le striatum dans le parkinsonisme et les constatations à la tomographie par l'émission de positons du transporteur de la dopamine (DAT-scan). Méthode: Des patients atteints de parkinsonisme, qui ont subi une imagerie par résonance magnétique du cerveau et un DAT-scan, ont été inclus dans l'étude. L'état clinique a été évalué au moyen de l'échelle de Hoehn et Yahr (HY), du Miniexamen de l'état mental coréen (MMSE-C), du Montreal Cognitive Assessment (MoCA) test coréen et de la batterie rapide d'efficience frontale (BREF). L'EVRd a été évalué au moyen d'échelles semi-quantitative et quantitative dans chacun des trois segments du striatum (noyau caudé, putamen antérieur et putamen postérieur) et le résultat a été exprimé au moyen d'un score EVRd. Le DAT-scan a été évalué qualitativement dans chaque segment comme étant normal ou anormal. Pour chaque segment, l'EVRd et le DAT-scan étaient classifiés comme concordants ou discordants. Un segment concordant était défini par la présence d'un EVRd et d'un DAT-scan anormal [Taux de concordance (TC) = nombre de segments concordants/nombre de segments concordants et discordants]. Résultats: Onze patients ont été inclus dans l'étude. Nous n'avons pas observé de corrélation significative entre la présence d'un EVRd et un DAT-scan anormal. Le TC moyen était de 0,39. Le TC n'était corrélé significativement à aucun des scores aux échelles cliniques ou de neuroimagerie. Le score de l'EVRd était corrélé significativement aux résultats du MMSE-C, du MoCA-C et de la BREF ( $\mathrm{r}=-0,675$, $-0,847$ et $-0,868$ respectivement), mais ne l'était pas au stage de HY. Conclusion: Un EVRd dans le striatum ne jouait pas un rôle significatif sur l'innervation dopaminergique mise en évidence par le DAT-scan et contribuait peu au parkinsonisme clinique ; cependant il était corrélé à l'atteinte cognitive.

Keywords: Dilated Virchow-Robin Space, Dopamine Transporter, Positron Emission Tomography, Parkinsonism, Striatum, Frontal executive dysfunction.

doi:10.1017/cjn.2015.43

Can J Neurol Sci. 2015; 42: 248-254

Virchow-Robin space (VRS) is a subpial space surrounding the perforating arteries and arterioles of the brain, and dilated VRS (dVRS) appears as small and sharply delineated structures of cerebrospinal fluid signal intensity that follow the orientation of the perforating vessels and run perpendicular to the brain surface. $^{1-3}$ The presence of dVRS has been reported in both normal persons and those with various disorders such as hypertension, depression, and dementia but the clinical implications of dVRS remain controversial. $^{2}$
Because the striatum (STR) is a critical region in the pathogenesis of parkinsonism, many previous studies have explored the role of dVRS in STR on parkinsonian patients and have yielded

From the Department of Neurology (DL, TA); Department of Nuclear Medicine (IKH), College of Medicine, Kyung Hee University, Seoul, Korea

Received August 3, 2014. Final Revisions Submitted February 2, 2015

Correspondence to Tae-Beom Ahn, Department of Neurology, Kyung Hee University Hospital, 23 Kyungheedae-ro, Dongdaemun-gu, Seoul 130-872, Republic of Korea.

Email: ricash@hanmail.net 
conflicting results. ${ }^{4-10}$ Some authors suggest that dVRS is either a single pathologic feature or a clinical modifier in parkinsonian patients. ${ }^{7,8,11}$ The dopamine transporter (DaT) is abundantly expressed in the striatal terminals of dopaminergic neurons and is well correlated with striatal dopamine concentrations. ${ }^{12}$ Dopamine transporter positron emission tomography (DaT-PET) is a useful diagnostic tool to investigate the dopaminergic innervation of STR in patients with parkinsonism. ${ }^{13,14}$

Although DaT-PET alterations can result from structural lesions, such as an infarct to the STR, the relationship between dVRS and DaT-PET remains undetermined. ${ }^{15}$ There are two types of dVRS. Type $1 \mathrm{dVRS}$ presents with normal surrounding tissue, and type 2 dVRS presents with surrounding rarefication and abnormal gliosis. ${ }^{4}$ The role of dVRS on DaT-PET likely differs based on the type of dVRS.

Based on the hypothesis that dVRS plays a modifying role in parkinsonism, we aimed to investigate the influence of dVRS in STR on the clinical features and neuroimaging of parkinsonian patients.

\section{MethodS \\ Participants}

Patients were recruited from those who visited our clinic for evaluation of parkinsonism. The evaluation was done by standard protocol of our Parkinson registry, including neurological evaluation of parkinsonism, cognitive function testing and brain magnetic resonance imaging (MRI). Dopamine transporter-PET was done in those with an uncertain diagnosis. Because only a few patients exhibited dVRS in STR on their MRI, we included all patients from our registry that underwent both $1.5 \mathrm{~T}$ MRI and DaT-PET and exhibited dVRS on their MRI, regardless of clinical diagnosis.

Parkinsonism was defined as the presence of at least two of four cardinal signs (rest tremor, rigidity, bradykinesia, and loss of postural reflexes). Clinical diagnosis of Parkinson Disease (PD) was made using the UK Parkinson's Disease Society Brain Bank Clinical Diagnostic Criteria (UKPDS). ${ }^{16}$ Diagnosis of Multiple System Atrophy (MSA), Progressive Supranuclear Palsy (PSP), and Frontotemporal Dementia (FTD) were made using the consensus clinical criteria for each diagnosis. ${ }^{17-19} \mathrm{~A}$ diagnosis of Vascular Parkinsonism (VaP) was made in patients with symmetric parkinsonism with predominance in the lower body and in the absence of typical features suggestive of other parkinsonian disorders. ${ }^{10}$ Those with a history of stroke within one year from the time of diagnosis and those with new vascular lesions on brain MRI were excluded.

Demographic data and vascular risk factors, including hypertension, diabetes, hyperlipidemia, smoking, heart disease and history of stroke, were assessed using medical records. Motor and cognitive statuses were evaluated by Hoehn and Yahr stage (HY stage), Korean Mini-Mental Status Examination (K-MMSE), Korean version of Montreal Cognitive Assessment (MoCA-K), and Frontal Assessment Battery (FAB).

This study was approved by the Institutional Review Board of Kyung Hee University Hospital. All participants gave informed consent before the study.

\section{Neuroimaging}

Virchow-Robin space in STR was defined as the presence of small (less than three $\mathrm{mm}$ ) isointense signals with cerebrospinal fluid on both T1 and T2 weighted images. ${ }^{1-3}$ Dilated VirchowRobin space was evaluated on axial MRI slices in which both the caudate nucleus $(\mathrm{CN})$ and the putamen could be best visualized. Each STR was divided into three segments: $\mathrm{CN}$, anterior putamen (AP), and posterior putamen (PP). Anterior putamen and PP were separated by an imaginary line from the genu of internal capsule, which was perpendicular to the anterior-posterior commissure line. The severity of dVRS was assessed in each segment using a visual semi-quantitative scale (none $=0$, mild $=1$, moderate $=2$, and severe $=3$ ) and a quantitative scale by counting the actual number of dVRS [0 (none), 1-9=1 (mild), 10-19=2 (moderate), and $\geq 20=3$ (severe) $]^{2,20,21}$ The sum of the six segment scores generated the dVRS score.

Dopamine transporter-PET was performed using ${ }^{18}$ F-fluorinated N-3-fluoropropyl-2beta-carboxymethoxy-3-beta(4-iodophenyl) notropane ( ${ }^{18} \mathrm{~F}$-FPCIT), as reported previously. ${ }^{22}$ The DaT-PET findings in the six segments (CN, AP, and PP, bilaterally) were blindly evaluated by an expert in nuclear medicine (IKH). The findings on DaT-PET were qualitatively designated as normal $(=0)$ or abnormal $(=1)$ in each segment, and scores were then summed to generate a DaT-PET score.

Anterior-posterior gradient ( $\mathrm{APg}$ ) was assessed by comparing the severity of dVRS and DaT-PET abnormality (ab-DaT-PET) in the anterior and posterior segments. Anterior-posterior gradient was determined to be present if dVRS in the $\mathrm{CN}$ was less severe than in the putamen and if dVRS in the AP was not more severe than in the PP. With regards to DaT-PET, APg was present if the abnormality was absent in the $\mathrm{CN}$ and found in both the AP and PP or in only the PP.

The correlation between dVRS and ab-DaT-PET was designated in each segment as concordant if dVRS was present with ab-DaT-PET or as discordant if dVRS was either present with normal DaT-PET or absent with ab-DaT-PET. A concordant segment was assumed to represent pathologic dVRS in STR. Concordance rate $(\mathrm{CR})$ was calculated $[\mathrm{CR}=$ number of concordant segments/number of concordant and discordant segments] to assess its correlation with clinical and neuroimaging scales. Patients were divided into two groups according to the mean $\mathrm{CR}$ value (one with $\mathrm{CR} \geq$ mean, and the other with $\mathrm{CR}<$ mean).

Leukoaraiosis (LA) was defined as the presence of hyperintense lesions in the periventricular or subcortical regions on fluid-attenuated inversion recovery MRI sequences. We evaluated LA severity based on a visual rating scale (LA score) ${ }^{23}$ LA score was designated as mild (1-4) or severe (>4). We defined silent infarctions (SIs) as focal hyperintensities of three mm or larger on T2-weighted images (T2WIs) in patients without relevant history or neurologic deficit. ${ }^{24}$ SIs showed hypointensity on T1-weighted images. Cerebral microbleeds (CMBs) were defined as small (less than five $\mathrm{mm}$ in diameter), rounded, homogeneous, and hypointense signals on the gradient echo or T2-weighted images. We differentiated CMBs from perforating vessels and iron deposition by reviewing serial images. ${ }^{25}$

\section{Statistical Analysis}

Groups were compared using the Student's $t$ test, MannWhitney U test or Chi-square (Fisher's exact) test. Agreement between semi-quantitative and quantitative scales on dVRS was assessed using the intraclass correlation coefficient (ICC). Correlations between neuroimaging and clinical scales were 
Table 1: Clinical diagnosis and analysis of magnetic resonance imaging and dopamine transporter positron emission tomography

\begin{tabular}{|c|c|c|c|c|c|c|c|c|c|}
\hline \multirow[b]{2}{*}{ Case (Sex/Age) } & \multirow[b]{2}{*}{ Diagnosis } & \multirow[b]{2}{*}{ dVRS CN } & \multirow[b]{2}{*}{ dVRS AP } & \multirow[b]{2}{*}{ dVRS PP } & \multirow[b]{2}{*}{$\begin{array}{l}\text { DaT-PET } \\
\text { abnormality }\end{array}$} & \multirow[b]{2}{*}{$\mathbf{C R}$} & \multicolumn{2}{|c|}{ A-P gradient } & \multirow[b]{2}{*}{ Other findings in MRI (LA score) } \\
\hline & & & & & & & dVRS & DaT-PET & \\
\hline Case $1(\mathrm{~F} / 73)$ & FTD & $\begin{array}{l}\mathrm{R} \text { mild } \\
\mathrm{L} \text { severe }\end{array}$ & B severe & B severe & Normal & 0 & - & NA & Mild LA (2) \\
\hline Case $2(\mathrm{M} / 65)$ & PSP & None & L severe & L mild & B CN, AP, PP & 0.33 & - & - & Hydrocephalus, mild LA (4) \\
\hline Case 3 (F/71) & $\mathrm{VaP}$ & None & B mild & $\begin{array}{l}\mathrm{R} \text { severe } \\
\mathrm{L} \text { moderate }\end{array}$ & Normal & 0 & + & NA & Severe LA (12) \\
\hline Case 4 (F/72) & PD & L mild & $\begin{array}{l}\text { R mild } \\
\text { L severe }\end{array}$ & None & B CN, AP, PP & 0.50 & - & - & Lacunar infarction (R cerebellum) \\
\hline Case $5(\mathrm{~F} / 72)$ & $\mathrm{PD}$ & None & $\mathrm{L}$ moderate & B mild & R PP & 0.33 & - & + & Mild LA (3) \\
\hline Case $6(\mathrm{M} / 74)$ & MSA & None & None & B mild & L CN, AP, PP & 0.25 & + & - & Mild LA (4) \\
\hline Case $7(\mathrm{~F} / 70)$ & PD & $\mathrm{R}$ mild & R severe & $\begin{array}{l}\mathrm{R} \text { severe } \\
\mathrm{L} \text { moderate }\end{array}$ & B AP, PP & 0.50 & $\begin{array}{l}-(\mathrm{L}) \\
+(\mathrm{R})\end{array}$ & + & $\begin{array}{l}\text { Severe LA (9), multiple silent infarcts (B subcortex, } \\
\text { R thalamus) }\end{array}$ \\
\hline Case $8(\mathrm{M} / 77)^{*}$ & PD & None & $\mathrm{L}$ mild & $\mathrm{L}$ moderate & L PP & 0.50 & + & + & $\begin{array}{l}\text { Right middle cerebral artery infarction, } \\
\text { hydrocephalus, mild LA ( } 3 \text { ) }\end{array}$ \\
\hline Case $9(\mathrm{M} / 69)$ & $\mathrm{VaP}$ & B severe & $\begin{array}{l}\text { R severe } \\
\mathrm{L} \text { mild }\end{array}$ & $\begin{array}{l}\mathrm{R} \text { severe } \\
\mathrm{L} \text { moderate }\end{array}$ & $\begin{array}{l}\text { R CN, AP } \\
\text { L AP, PP }\end{array}$ & 0.17 & - & $\begin{array}{l}-(\mathrm{R}) \\
+(\mathrm{L}) \\
\end{array}$ & $\begin{array}{l}\text { Severe LA (10), hydrocephalus, multiple cerebral } \\
\text { microbleeds ( } \mathrm{L} \text { thalamus and cerebellum) }\end{array}$ \\
\hline Case $10(F / 65)$ & MSA & none & $\mathrm{L}$ mild & $B$ mild & B PP & 0.67 & + & + & Mild LA (2) \\
\hline Case $11(\mathrm{M} / 80)$ & $\mathrm{VaP}$ & B severe & B severe & B severe & $\mathrm{B} \mathrm{CN}, \mathrm{AP}, \mathrm{PP}$ & 1.00 & - & - & Severe LA (11), hydrocephalus \\
\hline
\end{tabular}

Abbreviations: dVRS, dilated Virchow-Robin Space; CN, caudate nucleus; AP, anterior putamen; PP, posterior putamen; DaT-PET, dopamine transporter positron emission tomography;

CR, concordance rate; A-P, anterior-posterior; MRI, magnetic resonance imaging; LA, leukoaraiosis; FTD, Frontotemporal Dementia; R, right; L, left; B, bilateral; NA, not applicable;

PSP, Progressive Supranuclear Palsy; VaP, Vascular Parkinsonism; PD, Parkinson Disease; MSA, Multiple System Atrophy

*In Case 8, right caudate nucleus and putamen was not evaluated due to adjacent cerebral infarction. 
Table 2: Comparison between the patients with concordance rate $\geq 0.39$ and $<0.39$

\begin{tabular}{|c|c|c|c|c|}
\hline & All $(n=11)$ & $\mathrm{CR} \geq 0.39(\mathrm{n}=5)$ & $\mathrm{CR}<0.39(\mathrm{n}=6)$ & $p$ value \\
\hline Age & $72(65-80)$ & $72.0(65.0-80.0)$ & $71.5(65-74)$ & 0.662 \\
\hline Sex (Male/Female) & $5 / 6$ & $3 / 2$ & $3 / 3$ & 1.000 \\
\hline HY stage & $3(0.5-3.0)$ & $2.50(1.00-3.00)$ & $1.25(0.50-3.00)$ & 0.329 \\
\hline K-MMSE & $18.5(13-29)$ & $20.5(15.0-25.0)$ & $17.5(13.0-29.0)$ & 1.000 \\
\hline MoCA-K & $14.5(7-26)$ & $14.5(10.0-26.0)$ & $15.5(7.0-26.0)$ & 0.914 \\
\hline FAB & $9(3-15)$ & $7.0(6.0-9.0)$ & $10.5(3.0-15.0)$ & 0.548 \\
\hline DaT-PET (Normal/Abnormal) & $2 / 9$ & $0 / 5$ & $2 / 4$ & 0.455 \\
\hline DaT-PET score & $3.5(2.0-18.0)$ & $5.0(2.0-6.0)$ & $2.0(0-6.0)$ & 0.158 \\
\hline dVRS score & $6(2-18)$ & $8.0(3.0-18.0)$ & $5.5(2.0-15.0)$ & 0.762 \\
\hline LA score & $4(1-12)$ & $5.5(1.0-11)$ & $4.0(2.0-12.0)$ & 0.610 \\
\hline
\end{tabular}

Data expressed by median (range)

Abbreviations: CR, concordance rate; HY stage, Hoehn and Yahr stage; K-MMSE, Korean mini mental status examination; MoCA-K, Montreal Cognitive Assessment Korea; FAB, frontal assessment battery; DaT-PET, dopamine transporter positron emission tomography; dVRS, dilated Virchow-Robin

Space; LA, leukoaraiosis

analyzed by Spearman's rho. The cutoff $p$ value was set at 0.05 . All statistical analyses were conducted using the SPSS 19.0 package for Windows (IBM Corp. Armonk, NY, USA).

\section{RESULTS}

Eleven patients were included in this study. Clinical diagnoses and major neuroimaging findings are summarized in Table 1. The mean CR was 0.39. Demographics, clinical scales and neuroimaging findings are compared between those with $\mathrm{CR}$ $\geq 0.39$ and $\mathrm{CR}<0.39$ (Table 2).

Representative concordant and discordant cases are shown in Figure 1. We observed dVRS in 42 of 63 segments and ab-DaT-PET in 33 of 63 segments. Three segments from case eight were excluded from the analysis due to a lesion associated with adjacent ischemic stroke (Table 1).

Anterior-posterior gradient of dVRS and ab-DaT-PET was present in five cases (nine sides; Table 1). Anterior-posterior gradient
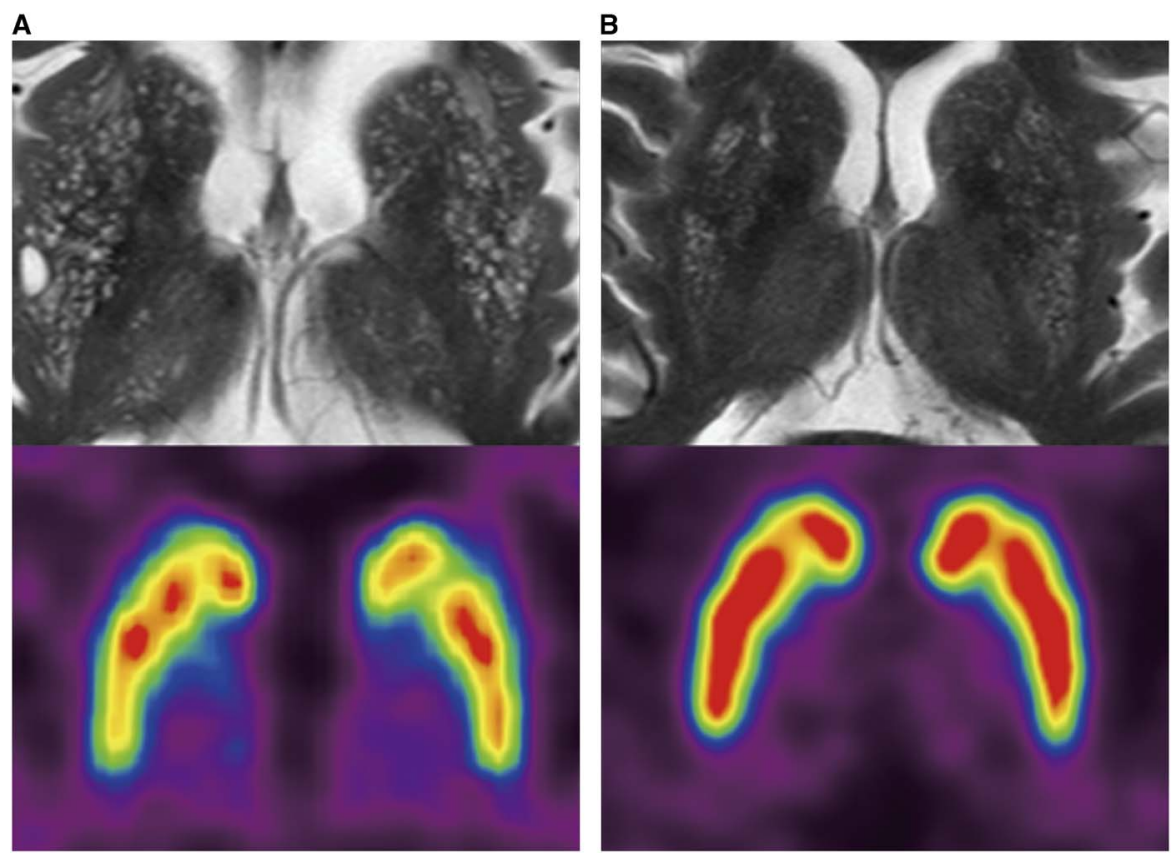

Figure 1: Concordance between dopamine transporter scan (DaT-PET) abnormality and dilated Virchow-Robin space (dVRS).

(A) A concordant case. DaT-PET shows abnormal findings and brain magnetic resonance imaging (MRI) shows moderate to severe degree of $d V R S$ in the bilateral caudate nucleus, anterior and posterior putamen. (B) A discordant case. DaT-PET shows normal findings, whereas brain MRI shows variable dVRS in the caudate nucleus and putamen. 
Table 3: Correlation coefficient between concordance rate and clinical or neuroimaging scales

\begin{tabular}{l|c|c|c|c|c|r|r}
\hline & HY stage & FAB & K-MMSE & MoCA-K & DaT-PET score & dVRS score & LA score \\
\hline $\mathrm{R}$ & 0.426 & 0.056 & 0.211 & 0.284 & 0.620 & -0.058 & -0.160 \\
\hline$p$ value & 0.192 & 0.887 & 0.559 & 0.427 & 0.056 & 0.873 & 0.659 \\
\hline
\end{tabular}

Abbreviations: R, correlation coefficient; HY stage, Hoehn and Yahr stage; K-MMSE, Korean mini mental status examination; MoCA-K, Montreal Cognitive Assessment Korea; FAB, frontal assessment battery; DaT-PET, dopamine transporter positron emission tomography; dVRS, dilated Virchow-Robin Space; LA, leukoaraiosis

was common in those with PD or MSA. In one case with PD and another with MSA, APg of dVRS was concordant with that of ab-DaT-PET. In VaP, there was only one case (two sides) with APg of dVRS and another (one side) with APg of ab-DaT-PET.

The conformity between scoring scales for dVRS was excellent $(\mathrm{ICC}=0.964, p$ value $<0.001)$. The quantitative $\mathrm{dVRS}$ scale was evaluated on only one axial slice because the same dVRS could be counted repeatedly in more than one slice. In addition, an accurate count of dVRS could be limited by imaginary segmentation of STR. Thus, we used a visual semi-quantitative scale for further statistical analysis.

There was no significant correlation between the presence of dVRS and ab-DaT-PET. No neuroimaging or clinical scales were significantly correlated with CR (Table 3 ). There were no significant differences in clinical features and neuroimaging findings between those patients with $\mathrm{CR} \geq 0.39$ and $\mathrm{CR}<0.39$ (Table 2). Leukoaraiosis, mild hydrocephalus, CMBs, and cerebral infarctions were variably present in brain MRI scans of certain individuals (Table 2). The dVRS score was strongly correlated with MoCA-K and FAB and moderately correlated with K-MMSE; however, it was not correlated with age, LA score, or HY stage (Figure 2).

\section{DISCUSSION}

In our study, we observed the presence of dVRS in various parkinsonian disorders, including PD. Although PD is considered to be a presynaptic disorder that leaves the STR least affected, in a pathologic study, 18 of $76(23.7 \%)$ patients with pathologically
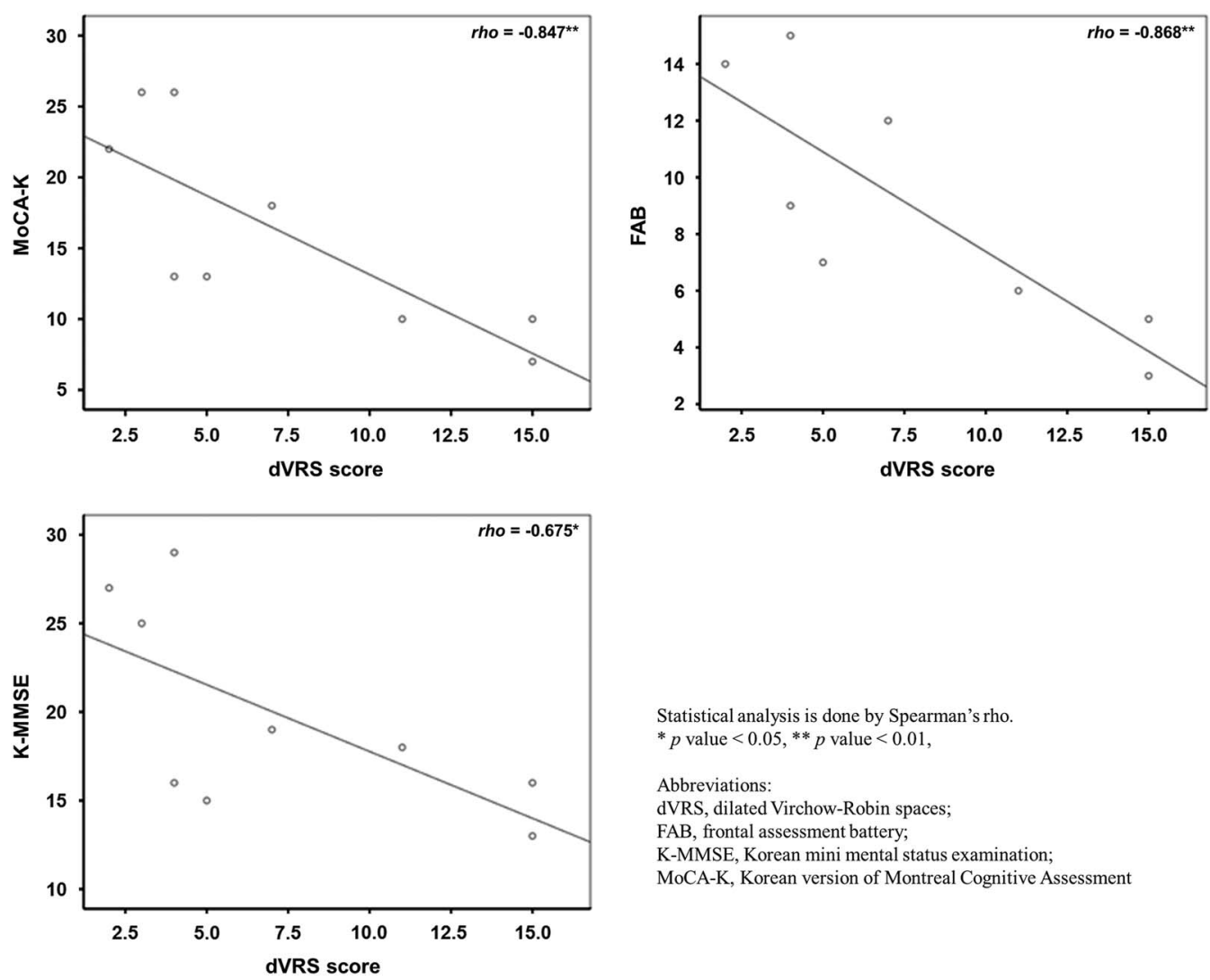

Statistical analysis is done by Spearman's rho.

$* p$ value $<0.05$, ** $p$ value $<0.01$

Abbreviations:

dVRS, dilated Virchow-Robin spaces;

$\mathrm{FAB}$, frontal assessment battery;

K-MMSE, Korean mini mental status examination;

MoCA-K, Korean version of Montreal Cognitive Assessment

Figure 2: Correlations between dilated Virchow Robin space (dVRS) score and clinical and neuroimaging findings. There is strong correlation between the dVRS score and Frontal Assessment Battery (FAB) and between the dVRS score and Korean version of Montreal Cognitive Assessment (MoCA-K), and moderate correlation between the dVRS score and Korean Mini-Mental Status Examination (K-MMSE). 
confirmed PD and 3 of 24 cases (12.5\%) with other etiologies had striatal pathology, including dVRS; however, the clinical implications of dVRS are obscure. ${ }^{6}$

In a previous study, the globus pallidus ipsilateral to the limbs exhibiting more severe motor symptoms showed more dVRS, suggesting a pallidotomy-like effect of dVRS on the contralateral limbs. ${ }^{8}$ There was a pathology-proven case of axial-dominant parkinsonism with isolated dVRS in STR that showed no response to levodopa. ${ }^{7}$ These studies suggested that dVRS could affect parkinsonian symptoms. However, in a recent case series of PD with large dVRS, dVRS was not associated with ab-DaT-PET but was correlated with atypical clinical features. ${ }^{11}$ In our study, the severity of parkinsonism, evaluated by HY stage, was not correlated with the dVRS score, excluding a significant role of dVRS in clinical parkinsonism.

Because type $2 \mathrm{dVRS}$ is associated with greater pathologic change, type 2 was suggested to be associated with clinical parkinsonism, whereas type 1 was a feature of normal aging. ${ }^{4,5,7}$ Recently, dVRS was suggested to be a marker of inflammation. ${ }^{26}$ Thus, type 2 dVRS may affect dopaminergic terminals in STR, resulting in decreased ${ }^{18}$ F-FPCIT uptake in DaT-PET, while type 1 dVRS may simply push dopaminergic terminals aside, leaving ${ }^{18} \mathrm{~F}$ FPCIT uptake unaffected. To test this hypothesis, we studied the relationship between dVRS and ab-DaT-PET. First, the correlation between the presence of dVRS and ab-DaT-PET was studied, which was found to be insignificant. Second, we studied whether APg of dVRS coincided with APg of ab-DaT-PET. Concurrence between APg of dVRS and ab-DaT-PET was observed in only two (three sides) out of four patients (eight sides) with PD, suggesting a minimal pathologic contribution of dVRS to ab-DaT-PET. Moreover, CR, a representation of type $2 \mathrm{dVRS}$, was not correlated with clinical parkinsonism. Thus, all the results showed that dVRS did not play an important role in ab-DaT-PET and clinical parkinsonism, suggesting that the majority of observed dVRS may be type 1 .

Dilated VRS severity was reported to be correlated with cognitive dysfunction. ${ }^{2}$ Our study also showed a significant correlation between dVRS and cognitive function tests. Frontal function was shown to be best correlated with dVRS severity (Spearman's rho between FAB and the dVRS score $=-0.868$, $p<0.01$ ). Our results reaffirm the role of STR in frontal function via the frontal-STR loop. ${ }^{27}$

In this study, we demonstrated the universality of dVRS in various parkinsonian disorders, the discrepancy between dVRS and ab-DaT-PET, and the possible cognitive contribution of dVRS. Our study is limited by the small number of subjects, heterogeneous clinical diagnoses and the inability to discriminate between two types of dVRS. Further studies using more sophisticated MRI techniques in a larger number of patients are necessary for a more comprehensive understanding of the role of dVRS.

\section{Disclosures}

Dokyung Lee does not have anything to disclose. Il Ki Hong does not have anything to disclose. Tae-Beom Ahn does not have anything to disclose.

\section{REFERENCES}

1. Heier LA, Bauer CJ, Schwartz L, Zimmerman RD, Morgello S, Deck MD. Large virchow-robin spaces: MR-clinical correlation. AJNR Am J Neuroradiol. 1989;10:929-36.
2. Maclullich AM, Wardlaw JM, Ferguson KJ, Starr JM, Seckl JR, Deary IJ. Enlarged perivascular spaces are associated with cognitive function in healthy elderly men. J Neurol Neurosurg Psychiatry. 2004;75:1519-23.

3. Udaka F, Sawada H, Kameyama M. White matter lesions and dementia: MRI-pathological correlation. Ann N Y Acad Sci. 2002;977:411-5.

4. Mancardi GL, Romagnoli P, Tassinari T, Gandolfo C, Primavera A, Loeb C. Lacunae and cribriform cavities of the brain. Correlations with pseudobulbar palsy and parkinsonism. Eur Neurol. 1988;28:11-7.

5. Murrow RW, Schweiger GD, Kepes JJ, Koller WC. Parkinsonism due to a basal ganglia lacunar state: Clinicopathologic correlation. Neurology. 1990;40:897-900.

6. Hughes AJ, Daniel SE, Kilford L, Lees AJ. Accuracy of clinical diagnosis of idiopathic parkinson's disease: A clinicopathological study of 100 cases. J Neurol Neurosurg Psychiatry. 1992;55:181-4.

7. Fenelon G, Gray F, Wallays C, Poirier J, Guillard A. Parkinsonism and dilatation of the perivascular spaces (etat crible) of the striatum: A clinical, magnetic resonance imaging, and pathological study. Mov Disord. 1995;10:754-60.

8. Laitinen LV, Chudy D, Tengvar M, Hariz MI, Bergenheim AT. Dilated perivascular spaces in the putamen and pallidum in patients with parkinson's disease scheduled for pallidotomy: A comparison between MRI findings and clinical symptoms and signs. Mov Disord. 2000;15:1139-44.

9. Duker AP, Espay AJ. Parkinsonism associated with striatal perivascular space dilation. Neurology. 2007;68:1540.

10. Zijlmans JC, Daniel SE, Hughes AJ, Revesz T, Lees AJ. Clinicopathological investigation of vascular parkinsonism, including clinical criteria for diagnosis. Mov Disord. 2004;19: 630-40.

11. Mestre TA, Armstrong MJ, Walsh R, et al. Can isolated enlarged virchow-robin spaces influence the clinical manifestations of parkinson's disease? Movement Disorders Clin Prac. 2014; 1:67-9.

12. Hersch SM, Yi H, Heilman CJ, Edwards RH, Levey AI. Subcellular localization and molecular topology of the dopamine transporter in the striatum and substantia nigra. J Comp Neurol. 1997;388:211-27.

13. Rinne JO, Ruottinen H, Bergman J, Haaparanta M, Sonninen P, Solin O. Usefulness of a dopamine transporter PET ligand [(18)F] beta-CFT in assessing disability in parkinson's disease. J Neurol Neurosurg Psychiatry. 1999;67:737-41.

14. Frost JJ, Rosier AJ, Reich SG, et al. Positron emission tomographic imaging of the dopamine transporter with 11C-WIN 35,428 reveals marked declines in mild parkinson's disease. Ann Neurol. 1993;34:423-31.

15. Vaamonde J, Flores JM, Gallardo MJ, Ibanez R. Subacute hemicorporal parkinsonism in 5 patients with infarcts of the basal ganglia. J Neural Transm. 2007;114:1463-7.

16. Gibb WR, Lees AJ. The relevance of the lewy body to the pathogenesis of idiopathic parkinson's disease. J Neurol Neurosurg Psychiatry. 1988;51:745-52.

17. Gilman S, Wenning GK, Low PA, et al. Second consensus statement on the diagnosis of multiple system atrophy. Neurology. 2008;71:670-6

18. Litvan I, Agid Y, Calne D, et al. Clinical research criteria for the diagnosis of progressive supranuclear palsy (steele-richardsonolszewski syndrome): Report of the NINDS-SPSP international workshop. Neurology. 1996;47:1-9.

19. Neary D, Snowden JS, Gustafson L, et al. Frontotemporal lobar degeneration: A consensus on clinical diagnostic criteria. Neurology. 1998;51:1546-54.

20. Doubal FN, MacLullich AM, Ferguson KJ, Dennis MS, Wardlaw JM. Enlarged perivascular spaces on MRI are a feature of cerebral small vessel disease. Stroke. 2010;41:450-4.

21. Klarenbeek P, van Oostenbrugge RJ, Lodder J, Rouhl RP, Knottnerus IL, Staals J. Higher ambulatory blood pressure relates to enlarged virchow-robin spaces in first-ever lacunar stroke patients. J Neurol. 2013;260:115-21. 
22. Hwang KJ, Hong IK, Ahn TB, Yi SH, Lee D, Kim DY. Cortical hemichorea-hemiballism. J Neurol. 2013;260:2986-92.

23. Wahlund LO, Barkhof F, Fazekas F, et al. European Task Force on Age-Related White Matter Changes. A new rating scale for agerelated white matter changes applicable to MRI and CT. Stroke. 2001;32:1318-22.

24. Zhu YC, Dufouil C, Tzourio C, Chabriat H. Silent brain infarcts: A review of MRI diagnostic criteria. Stroke. 2011;42:1140-5.
25. Greenberg SM, Vernooij MW, Cordonnier C, et al. Microbleed Study Group. Cerebral microbleeds: A guide to detection and interpretation. Lancet Neurol. 2009;8:165-74.

26. Wuerfel J, Haertle M, Waiczies H, et al. Perivascular spaces-MRI marker of inflammatory activity in the brain? Brain. 2008;131:2332-40.

27. Alexander GE, DeLong MR, Strick PL. Parallel organization of functionally segregated circuits linking basal ganglia and cortex. Annu Rev Neurosci. 1986;9:357-81. 